\title{
Colored Partitions and the Fibonacci Sequence
}

J.P.O. SANTOS ${ }^{1}$, M. IVKOVIĆ ${ }^{2}$ IMECC, UNICAMP, Cx.P. 6065, 13083-970 Campinas, SP, Brazil.

\begin{abstract}
We present interesting combinatorial interpretations for the Fibonacci numbers in terms of colored partitions obtained by using finite versions of two identities of the Rogers-Ramanujan type. New formula for the Fibonacci numbers is also given.
\end{abstract}

Key words: Partitions, Fibonacci numbers, Rogers-Ramanujan identities.

Classification: Primary 11P81; Secondary 11B39.

\section{Introduction}

Considered for the first time in a modest example for the facility of calculation in positional number system (Liber abaci 1202.), the Fibonacci numbers showed to be intrinsic in nature (phyllotaxis), and omnipresent in arts (poetry, architecture, etc.).

Many properties of these numbers are known. They appear in numerical mathematics (Adby [1]), game theory (Tosić [14]), as well as in combinatorics and partition theory, where there are interpretations in terms of compositions, for example:

The number of compositions of $n$ in which no 1's appear is $F_{n-1}$ (Andrews [2]).

Or in terms of partitions, one interpretation obtained by the authors is:

The total number of partitions into at most $N$ parts in which every even smaller than the largest part appears at least once is equal to $F_{2 N+2}$ (Santos \& Ivković [10]).

In this paper we present two new combinatorial interpretations in terms of colored partitions based on identities number 63 and 62 in the Slater's list of identities of the Rogers-Ramanujan type [13]. We use a method introduced by Andrews [5], and used by Santos [9] to obtain finite versions of Rogers-Ramanujan type identities. Basic tools are presented in the following section.

\footnotetext{
${ }^{1}$ josepli@ime.unicamp.br

2 milos@ime.unicamp.br
} 


\section{Basic definitions}

We start with Gaussian polynomials (see Andrews [2]), that are q-analog of the binomial coefficients:

$$
\left[\begin{array}{c}
n \\
m
\end{array}\right]= \begin{cases}\frac{(q)_{n}}{(q)_{m}(q)_{n-m}}, & \text { if } 0 \leq m \leq n \\
0, & \text { otherwise }\end{cases}
$$

where

$$
(a)_{n}=(a ; q)_{n}=(1-a)(1-a q) \ldots\left(1-a q^{n-1}\right)
$$

$n$ a nonnegative integer. We define also

$$
(a)_{\infty}=\lim _{k \rightarrow \infty}(a ; q)_{k} .
$$

When dealing with the expression

$$
\left(1+x+x^{2}\right)^{n}
$$

we call the coefficients of $x^{j}$ in the expanded form of (2.2) the trinomial coefficients.

It is easy to show that if

$$
\left(1+x+x^{2}\right)^{n}=\sum_{j=-n}^{n}\left(\begin{array}{l}
n \\
j
\end{array}\right)_{2} x^{j+n}
$$

then

$$
\begin{aligned}
\left(\begin{array}{l}
n \\
j
\end{array}\right)_{2} & =\sum_{h \geq 0} \frac{n !}{h !(h+j) !(n-j-2 h) !} \\
& =\sum_{h \geq 0}(-1)^{h}\left(\begin{array}{l}
n \\
h
\end{array}\right)\left(\begin{array}{c}
2 n-2 h \\
n-j-h
\end{array}\right)
\end{aligned}
$$

also

$$
\left(\begin{array}{l}
n \\
j
\end{array}\right)_{2}=\left(\begin{array}{c}
n \\
-j
\end{array}\right)_{2}
$$

and

$$
\left(\begin{array}{l}
n \\
j
\end{array}\right)_{2}=\left(\begin{array}{l}
n-1 \\
j-1
\end{array}\right)_{2}+\left(\begin{array}{c}
n-1 \\
j
\end{array}\right)_{2}+\left(\begin{array}{c}
n-1 \\
j+1
\end{array}\right)_{2} .
$$

The following expressions (Andrews \& Baxter [7]) are $q$-analogs of the trinomial coefficient in the same way that the Gaussian polynomial is a $q$-analog of the binomial coefficient, that is, the limit of each one of them when $q$ approaches 1 is equal to the trinomial coefficient given by (2.4) and (2.5).

$$
\begin{aligned}
& T_{0}(m, A, q)=\sum_{j=0}^{m}(-1)^{j}\left[\begin{array}{c}
m \\
j
\end{array}\right]_{q^{2}}\left[\begin{array}{c}
2 m-2 j \\
m-A-j
\end{array}\right], \\
& T_{1}(m, A, q)=\sum_{j=0}^{m}(-q)^{j}\left[\begin{array}{c}
m \\
j
\end{array}\right]_{q^{2}}\left[\begin{array}{c}
2 m-2 j \\
m-A-j
\end{array}\right] .
\end{aligned}
$$


There are the following Pascal-triangle type relations:

$$
\begin{aligned}
T_{1}(m, A, q)= & T_{1}(m-1, A, q)+q^{m+A_{1}} T_{0}(m-1, A+1, q) \\
& +q^{m-A} T_{0}(m-1, A-1, q), \\
T_{0}(m, A, q)= & T_{0}(m-1, A-1, q)+q^{m+A} T_{1}(m-1, A, q) \\
& +q^{2 m+2 A} T_{0}(m-1, A+1, q) .
\end{aligned}
$$

It is also valid (Andrews \& Baxter [7]):

$$
\begin{aligned}
& T_{1}(m, A, q)-q^{m-A} T_{0}(m, A, q)-T_{1}(m, A+1, q) \\
& +q^{m+A+1} T_{0}(m, A+1, q)=0 .
\end{aligned}
$$

From (2.8) and (2.9) we can see that

$$
\begin{aligned}
& T_{0}(m, A, q)=T_{0}(m,-A, q), \\
& T_{1}(m, A, q)=T_{1}(m,-A, q),
\end{aligned}
$$

where we have used the following property of the Gaussian polynomials which follows from the definition (2.1):

$$
\left[\begin{array}{l}
n \\
m
\end{array}\right]=\left[\begin{array}{c}
n \\
n-m
\end{array}\right]
$$

\section{The Main theorem}

\subsection{Identity number 63}

We start this section by considering identity number 63 in the Slater's list [13] that is

$$
\left(q ; q^{5}\right)_{\infty}\left(q^{4} ; q^{5}\right)_{\infty}\left(q ; q^{10}\right)_{\infty}\left(q^{9} ; q^{10}\right)_{\infty}(-q ; q)_{\infty}=\sum_{n=0}^{\infty} \frac{(-q ; q)_{n} q^{\frac{3}{2} n(n+1)}}{(q ; q)_{2 n}}
$$

On the left side we introduce a new variable " $t$ " in the following way:

$$
\begin{aligned}
f_{63}(t, q) & =\sum_{n=0}^{\infty} \frac{(-t ; q)_{n+1} t^{3 n} q^{\frac{3}{2} n(n+1)}}{\left(t^{2} ; q\right)_{2 n+1}} \\
& =\sum_{n=0}^{\infty} \frac{(-t q ; q)_{n} t^{3 n} q^{\frac{3}{2} n(n+1)}}{(1-t)\left(t^{2} q ; q\right)_{2 n}}
\end{aligned}
$$

in this sum the factor

$$
(1+t q) \ldots\left(1+t q^{n}\right) t^{n} q^{1+2+\ldots+n}
$$


is clearly the generating function for partitions where every integer less then or equal to the largest part appears at least once and at most twice. We shall call them the green parts.

The factor

$$
\frac{t^{2 n} q^{2+4+\ldots+2 n}}{(1-t)\left(1-t^{2} q\right) \ldots\left(1-t^{2} q^{2 n+1}\right)}
$$

is the generating function for partitions where every even part less than or equal to the largest part appears at least once. These are the yellow parts.

Notice that the largest yellow part that is even is equal to twice the largest green part.

Now we return to $(3.2)$. It is valid

$$
(1-t)\left(1-t^{2} q\right) f_{63}(q, t)=1+t^{3} q^{3} f_{63}(q, t q)
$$

Taking $f_{63}(t, q)=\sum_{n=0}^{\infty} P_{n}(q) t^{n}$ from (3.6) we have

$$
\begin{array}{r}
P_{0}=P_{1}=1 ; \quad P_{2}=1+q \\
P_{n}=P_{n-1}+q P_{n-2}-\left(q-q^{n}\right) P_{n-3} .
\end{array}
$$

Santos in [8] conjectured:

$$
P_{n}=\sum_{j=-\infty}^{\infty} q^{\frac{15}{2} j^{2}+\frac{7}{2} j} T_{1}\left(n, 5 j+1, q^{\frac{1}{2}}\right)-\sum_{j=-\infty}^{\infty} q^{\frac{15}{2} j^{2}+\frac{13}{2} j+1} T_{1}\left(n, 5 j+2, q^{\frac{1}{2}}\right) .
$$

We prove this in the appendix.

Once having proved this theorem, we can make a connection between the greenyellow partitions and the Fibonacci numbers. Taking $q \rightarrow 1$ in (3.7) recurrent relation for the Fibonacci numbers appears. Thus, it is valid:

Theorem 1. The total number of green-yellow partitions in which the number of green parts plus twice the number of yellow parts is smaller then or equal to $N$ is equal to $F_{n+1}$.

Here, of course $F_{n}$ is the n-th Fibonacci number. Considering that $T_{1}$ is a qanalog of the trinomial coefficients we may take the limit in (3.8) when $q$ approaches 1, getting a formula for the Fibonacci numbers:

$$
F_{N}=\sum_{j=-\infty}^{\infty}\left(\left(\begin{array}{c}
N \\
5 j+1
\end{array}\right)_{2}-\left(\begin{array}{c}
N \\
5 j+2
\end{array}\right)_{2}\right)
$$




\subsection{Identity number 62}

Identity number 62 in the Slater's list [13] is very similar:

$$
\left(q^{2} ; q^{5}\right)_{\infty}\left(q^{3} ; q^{5}\right)_{\infty}\left(q^{3} ; q^{10}\right)_{\infty}\left(q^{7} ; q^{10}\right)_{\infty}(-q ; q)_{\infty}=\sum_{n=0}^{\infty} \frac{(-q ; q)_{n} q^{\frac{1}{2} n(3 n+1)}}{(q ; q)_{2 n}}
$$

The new parameter " $t$ " is introduced in the similar way:

$$
f_{62}(t, q)=\sum_{n=0}^{\infty} \frac{(-t ; q)_{n+1} t^{3 n} q^{\frac{1}{2} n(3 n+1)}}{\left(t^{2} ; q^{2}\right)_{2 n+1}} .
$$

The only difference is that a generating function for the yellow parts now is:

$$
\frac{t^{2 n} q^{1+3+\ldots+2 n-1}}{(1-t)\left(1-t^{2} q\right) \ldots\left(1-t^{2} q^{2 n+1}\right)}
$$

i.e., generating function for the partitions where every odd integer less than or equal to the largest part appears at least once.

Bijection between the partitions of the yellow-green type connected with identity 63 and those connected with identity 62 is direct.

Not surprisingly, when writing $f_{62}$ in a form $\sum_{n=-\infty}^{\infty} Q_{n} t^{n}$ polynomials $Q_{n}$ satisfy very similar recurrent relation as $P_{n}$ :

$$
\begin{array}{r}
Q_{0}=Q_{1}=1 ; \quad Q_{2}=1+q ; \\
Q_{n}=Q_{n-1}+q Q_{n-2}-\left(q-q^{n-1}\right) Q_{n-3} .
\end{array}
$$

Explicit formula for this family is

$$
Q_{n}=\sum_{j=-\infty}^{\infty} q^{\frac{15}{2} j^{2}+\frac{1}{2} j} Q_{1}\left(n, 5 j, q^{\frac{1}{2}}\right)-\sum_{j=-\infty}^{\infty} q^{\frac{15}{2} j^{2}+\frac{11}{2} j+1} Q_{1}\left(n, 5 j+2, q^{\frac{1}{2}}\right) .
$$

Giving, at the end, the same formula (3.9) for the Fibonacci numbers.

Observation: Identities 62 and 63 from the Slater's list are equal to the identities number 46 and 44 respectively.

\section{Appendix}

Theorem 2. The family of polynomials defined in (3.8) satisfies recurrent relation $(3.7)$. 
Proof: To make calculation easier the base is changed by taking $q \rightarrow q^{2}$. We need to prove

$$
\begin{aligned}
& \sum_{j=-\infty}^{\infty} q^{15 j^{2}+7 j} T_{1}(n, 5 j+1, q)-\sum_{j=-\infty}^{\infty} q^{15 j^{2}+13 j+1} T_{1}(n, 5 j+2, q) \\
& =\sum_{j=-\infty}^{\infty} q^{15 j^{2}+7 j} T_{1}(n-1,5 j+1, q)-\sum_{j=-\infty}^{\infty} q^{15 j^{2}+13 j+1} T_{1}(n-1,5 j+2, q) \\
& +\sum_{j=-\infty}^{\infty} q^{15 j^{2}+7 j+1} T_{1}(n-2,5 j+1, q)-\sum_{j=-\infty}^{\infty} q^{15 j^{2}+13 j+2} T_{1}(n-2,5 j+2, q) \\
& +\left(q^{2 n}-q^{2}\right)\left(\sum_{j=-\infty}^{\infty} q^{15 j^{2}+7 j} T_{1}(n-3,5 j+1, q)-\sum_{j=-\infty}^{\infty} q^{15 j^{2}+13 j+1} T_{1}(n-3,5 j+2, q)\right) .
\end{aligned}
$$

From now on all the polynomials are in base " $q$ ", so we omit to explicitly state it, i.e., $T_{0}(n-1,5 j+2)=T_{0}(n-1,5 j+2, q)$. After applying (2.10) on both sums on the left and some cancellations, the expression is

$$
\begin{aligned}
& \sum_{j=-\infty}^{\infty} q^{15 j^{2}+12 j+n+1} T_{0}(n-1,5 j+2)+\sum_{j=-\infty}^{\infty} q^{15 j^{2}+2 j+n-1} T_{0}(n-1,5 j) \\
& -\sum_{j=-\infty}^{\infty} q^{15 j^{2}+18 j+4+n} T_{0}(n-1,5 j+3)-\sum_{j=-\infty}^{\infty} q^{15 j^{2}+8 j+n} T_{0}(n-1,5 j+1) \\
& =\sum_{j=-\infty}^{\infty} q^{15 j^{2}+7 j+1} T_{1}(n-2,5 j+1)-\sum_{j=-\infty}^{\infty} q^{15 j^{2}+13 j+2} T_{1}(n-2,5 j+2) \\
& +\left(q^{2 n}-q^{2}\right)\left(\sum_{j=-\infty}^{\infty} q^{15 j^{2}+7 j} T_{1}(n-3,5 j+1)-\sum_{j=-\infty}^{\infty} q^{15 j^{2}+13 j+1} T_{1}(n-3,5 j+2)\right) .
\end{aligned}
$$

By making " $j \rightarrow-j$ " and " $j \rightarrow j+1$ " at the first sum on the left that sum cancels with third by (2.13). The two sums left on left side we transform by (2.12) so they are written using $T_{1}$ polynomials. That side becomes

$$
\sum_{j=-\infty}^{\infty} q^{15 j^{2}+3 j} T_{1}(n-1,5 j)-\sum_{j=-\infty}^{\infty} q^{15 j^{2}+3 j} T_{1}(n-1,5 j) .
$$

Now we apply (2.10) on this two sums. Four resulting sums cancel by (2.12) leaving

$$
\sum_{j=-\infty}^{\infty} q^{15 j^{2}-2 j+n} T_{0}(n-2,5 j-1)-\sum_{j=-\infty}^{\infty} q^{15 j^{2}+8 j+n} T_{0}(n-2,5 j+2) .
$$

By (2.11) this two sums can be written as:

$$
\sum_{j=-\infty}^{\infty} q^{15 j^{2}+2 j+n-1} T_{0}(n-3,5 j)+\sum_{j=-\infty}^{\infty} q^{15 j^{2}+7 j+2 n} T_{1}(n-3,5 j+1)
$$




$$
\begin{aligned}
& +\sum_{j=-\infty}^{\infty} q^{15 j^{2}+12 j+3 n-3} T_{0}(n-3,5 j+3)-\sum_{j=-\infty}^{\infty} q^{15 j^{2}+8 j+n} T_{0}(n-3,5 j+1) \\
& -\sum_{j=-\infty}^{\infty} q^{15 j^{2}+13 j+2 n} T_{1}(n-3,5 j+2)-\sum_{j=-\infty}^{\infty} q^{15 j^{2}+2 j+n-1} T_{0}(n-3,5 j+3) .
\end{aligned}
$$

The second and the fourth sum from this expression cancel with corresponding sums on the right side of (A.1). The third and the sixth cancel between themselves.

Now we return to the right side. By applying (2.10) on the first two sides of (A.1) and some cancellations that side becomes:

$$
\begin{array}{r}
\sum_{j=-\infty}^{\infty} q^{15 j^{2}+12 j+n+1} T_{0}(n-3,5 j+2)+\sum_{j=-\infty}^{\infty} q^{15 j^{2}+2 j+n-1} T_{0}(n-3,5 j) \\
-\sum_{j=-\infty}^{\infty} q^{15 j^{2}+18 j+n+4} T_{0}(n-3,5 j+3)-\sum_{j=-\infty}^{\infty} q^{15 j^{2}+8 j+n} T_{0}(n-3,5 j+1) .
\end{array}
$$

The first sum cancels with the third. The second and the fourth cancel with the remaining sums from the left side, thus proving the identity.

\section{References}

[1] P.Rr Adby and M.A.H. Dempster, "Introduction to Optimization Methods", Chapman \& Hall, London, 1974.

[2] G.E. Andrews, "The Theory of Partitions", Encyclopedia of Mathematics and Its Applications (Rota, Editor), Vol. 2, G.-C, Addison-Wesley, Reading, 1976. (Reissued: Cambridge University Press, London and New York, 1985).

[3] G.E. Andrews, " $q$-Series: Their development and application in analysis, number theory, combinatorics, physics and computer algebra", CBMS Regional Conf. Ser. in Math., nํㅡ 66, Amer. Math. Soc., Providence, R.I., 1986.

[4] G.E. Andrews, Euler's "Exemplum Memorabile Inductionis Fallacis and qtrinomio coefficients", Dep. of Math. Penn State, Report \# PM 65, May 1989.

[5] G.E. Andrews, "Combinatorics and Ramanujan's 'lost' notebook", London Math. Soc. Lecture Note Series, No. 103, Cambridge Univ. Press, London, 1985, pp. 1-23.

[6] G.E. Andrews, q-Trinomial Coefficients and Rogers-Ramanujan Type Identities, in Analytic Number Theory (B. Berndt ed.), Boston, 1990.

[7] G.E. Andrews and R.J. Baxter, Lattice gas generalization of the hard hexagon model. III. q-trinomio coefficients, J. Stat. Phys., 47 (1987), 297-330. 
[8] J.P.O. Santos, "Computer algebra and identities of the Rogers-Ramanujan type". Ph.D. Thesis, Pennsylvania State University, 1991.

[9] J.P.O. Santos, On the combinatorics of polynomial generalizations of RogersRamanujan type identities, Discrete Mathematics, 254 No. 1-3 (2002), pp.497511.

[10] J.P.O. Santos and M. Ivković, Fibonacci Numbers and Partitions, Fibonacci Quarterly, 41 No. 3 (2003), 263-278.

[11] J.P.O. Santos and P. Mondek, Extending Theorems of Göllnitz, A New Family of Partition Identities, The Ramanujan Journal, 3 (1999), 359-365.

[12] J.P.O. Santos and A.V. Sills, q-Pell Sequences and Two Identities of V.S Lebesgue, Discrete Mathematics, 257, No. 1 (2002), 125-142.

[13] L.J. Slater, Further identities of the Rogers-Ramanujan type, Proc. London Math. Soc. 54, No. 2 (1952), 147-167.

[14] R. Tosić, Kombinatorika (in serbian). Univerzitet u Novom Sadu (1999), Novi Sad. 